\title{
BÀN VỀ ĐÁNH GIÁ THEO CÁC PHƯƠNG PHÁP KRIGING VÀ COLLOCATION
}

\author{
PGS. TSKH. HÀ MINH HOÀ \\ Viện Khoa học Đo đạc và Bản đồ
}

\section{Tóm tắt:}

Dựa trên các điều kiện không xê dịch và sai số trung phương cực tiểu của đánh giá nội suy các đại lượng ngẫu nhiên trong trường ngẫu nhiên theo lý thuyết địa thống kê, bài báo khoa học này đã xem xét các cơ sở khoa học của các phương pháp kriging, collocation và chỉ ra rằng lý thuyết của phương pháp collocation được xây dụ̣ng không dựa trên điều kiện không xê dịch. Với mục đích nhận được đánh giá sự không xê dịch của độ lệch xác suất theo phương pháp collocation, các giá trị gần đúng của đại lượng ngẫu nhiên xác suất nhất được nội suy phải được xác định từ chính các đại lượng ngẫu nhiên trong trường ngẫu nhiên. Từ các kết quả nghiên cứu, bài báo đã chỉ ra sự không thích hợp của việc sử dụng các dị thường trọng lực được xác định từ mô hình EGM2008 làm các giá trị gần đúng của các giá trị dị thường được nội suy dựa trên các giá trị dị thường trọng lực mặt đất ở Việt Nam do các dữ liệu trọng lực ở Việt Nam không tham gia tính toán các hệ số điều hòa của mô hình EGM2008.

\section{1. Đặt vấn đề}

Chúng ta ký hiệu $D$ là trường ngẫu nhiên. Giả thiết rằng trên tập hợp gồm điểm chúng ta đã xác định được $\mathrm{n}$ giá trị biến ngẫu nhiên $L\left[\chi_{i}\right](i=1,2, \ldots, n)$, ở đây $\chi_{i}$ là vectơ vị trí không gian của biến ngẫu nhiên $L\left[\chi_{i}\right]$ Bài toán được đặt ra là cần xác định biến ngẫu nhiên tin cậy nhất $\widetilde{L}\left(\chi_{P}\right)$ tại điểm $\mathrm{p}$ thuộc tập hợp $P^{e} \in D$ từ các tập hợp các biến ngẫu nhiên từ tập hợp $Q$ (xem hình 1 ở dưới đây) sao cho thỏa mãn các điều kiện:

- Không xê dịch

$$
E\left[\widetilde{L}\left(\chi_{P}\right)-\bar{L}\left(\chi_{P}\right)\right]=0,
$$

- Sai số trung phương cực tiểu

$$
E\left[\widetilde{L}\left(\chi_{P}\right)-\bar{L}\left(\chi_{P}\right)\right]^{2}=\min ,
$$

ở đây $\mathrm{E}[$.$] - ký hiệu kỳ vọng toán học, \bar{L}\left(\chi_{P}\right)$ là đánh giá gần đúng bất kỳ của biến ngẫu nhiên cần tìm $\widetilde{L}\left(\chi_{P}\right)$

Các điều kiện (1) và (2) tương ứng với trường ngẫu nhiên tĩnh tại theo cách tiếp cận Wiener - Kolgomorov, theo đó Wiener $\mathrm{N}$. đã xác định các điều kiện trên theo nguyên tắc tất định (deterministic principle) (xem Wiener N. (1949)), còn Kolgomorov A. N. theo nguyên tắc ngẫu nhiên (xem Kolmogorov A.N. (1933)).

Trên hình 1 ở dưới đây, tập hợp $Q$ là tập hợp các điểm được phân bố trên bề mặt Trái đất mà tại đó xác định được các đại lượng vật lý như dị thường trọng lực, dị thường độ cao v.v.., còn tập hợp $P^{e}$ là tập hợp các điểm mà các đại lượng vật lý trên các điểm này 
được xác định theo phương pháp nội suy từ các đại lượng vật lý trong tập hợp $Q$. Trong thực tế, tập hợp $P^{e}$ là mạng lưới (grid) bao gồm các ô chuẩn (cell) hình vuông (hoặc hình chữ nhật) được tạo bởi sự giao cắt của các đường vĩ tuyến (hoặc các đường tọa độ $\mathrm{y}$ trong hệ tọa độ phẳng Gauss, UTM) với các đường kinh tuyến (hoặc các đường tọa độ $\mathrm{x}$ trong hệ tọa độ phẳng Gauss, UTM). Điểm $p \in P^{e}$ là điểm giao cắt (hay còn được gọi là đỉnh của ô chuẩn) mà tại đó cần xác định đại lượng vật lý (dị thường trọng lực, dị thường độ cao .v.v...) từ các đại lượng vật lý trong tập hợp Q.

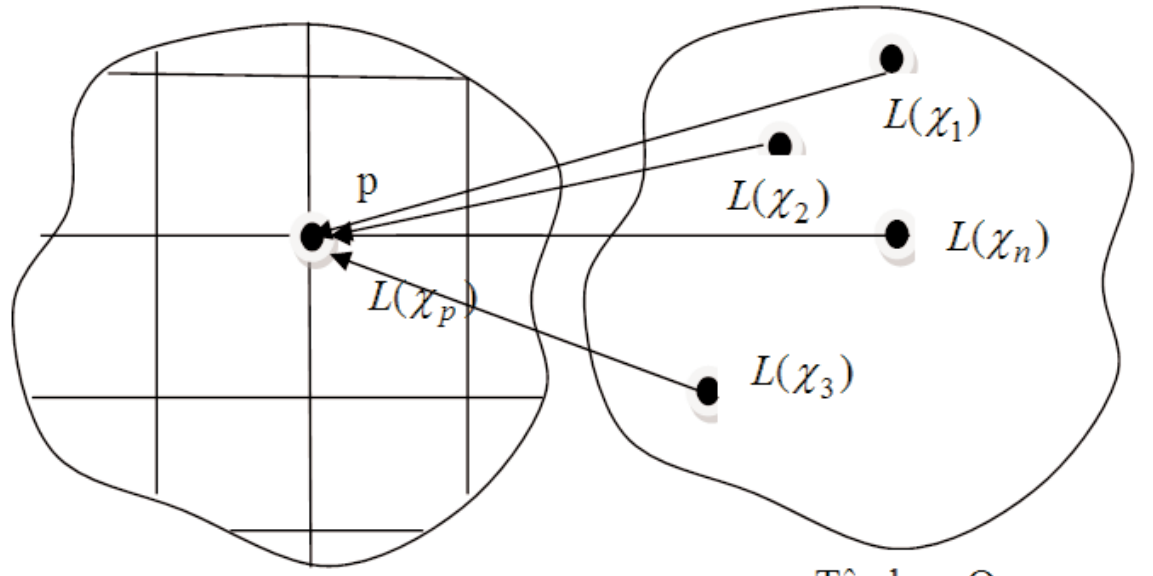

Tập hợp $P^{e}$ (Grid

Tập hợp Q

của CSDL)

Hình 1: Quan hệ giữa các tập hợp $Q$ và $P^{e}$ trong trường ngẫu nhiên $D$

Đại lượng $\bar{L}\left(\chi_{P}\right)$ trong các công thức (1) và (2) là đánh giá bất kỳ của biến ngẫu nhiên tại điểm $p \in P^{e}$ sao cho

$$
E\left[\bar{L}\left(\chi_{p}\right)\right]=X\left(\chi_{p}\right),
$$

Ở đây $X\left(\chi_{P}\right)$ là giá trị thực của biến ngẫu nhiên tại điểm $p \in P^{e}$

Với điều kiện (3) chúng ta thấy rằng các điều kiện (1) và (2) hoàn toàn tương ứng với định lý Gauss - Markov (xem trong Hà Minh Hòa (2014)). Thật vậy, lưu ý (3) từ điều kiện (1) suy ra $E\left[\widetilde{L}\left(\chi_{p}\right)\right]=X\left(\chi_{p}\right)$. Đây là điều kiện không xê dịch của biến ngẫu nhiên $\widetilde{L}\left(\chi_{P}\right)$ cần đánh giá trong định lý Gauss - Markov. Khi ký hiệu $V\left(\chi_{P}\right)=\widetilde{L}\left(\chi_{P}\right)-\bar{L}\left(\chi_{P}\right)$ là số hiệu chỉnh vào giá trị gần đúng $L\left(\chi_{p}\right)$ thì điều kiện (2) chính là điều kiện sai số trung phương cực tiểu của biến ngẫu nhiên $\widetilde{L}\left(\chi_{P}\right)$ cần đánh giá trong định lý Gauss - Markov. Như vậy, dựa trên định lý Gauss - Markov, để thỏa mãn các điều kiện (1) và (2), các biến ngẫu nhiên $L\left[\chi_{i}\right]$ (i $=1,2, \ldots ., n)$ trong tập hợp $Q$ không được chứa các sai số thô và các sai số hệ thống. Ngoài ra, như đã chứng minh trong tài liệu Hà Minh Hòa (2014), để thỏa mãn điều kiện (3), giá trị gần đúng $L\left(\chi_{p}\right)$ phải là đại lượng ngẫu nhiên, tức nó phải được tính toán từ các biến ngẫu nhiên $L\left[\chi_{i}\right](i=1,2, \ldots . ., n)$ trong tập hợp $Q$. Khi đó, từ hai điều kiện (1), (2) chúng ta thấy rằng giá trị tin cậy nhất $\widetilde{L}\left(\chi_{P}\right)$ của biến ngẫu nhiên tại điểm $\mathrm{p}$ thuộc tập hợp $P^{e} \in D$ phải thỏa mãn điều kiện không xê dịch, tức gần nhất với giá trị thực của nó, và có sai số 
trung phương nhỏ nhất, tức độ chính xác của nó cao nhất.

Chúng ta ký hiệu vectơ các biến ngẫu nhiên dưới dạng:

$$
L(\chi)=\left[\begin{array}{l}
L\left(\chi_{1}\right) \\
L\left(\chi_{2}\right) \\
\\
L\left(\chi_{n}\right)
\end{array}\right],
$$

có vectơ các giá trị trung bình xác suất

$$
E[L(\chi)]=\Omega(\chi)=\left[\begin{array}{l}
\mu\left(\chi_{1}\right) \\
\mu\left(\chi_{2}\right) \\
. \\
\mu\left(\chi_{n}\right)
\end{array}\right] .
$$

Trong lý thuyết địa thống kê, vectơ $\Omega(\chi)(4)$ được gọi là vectơ xu thế (trend) và nó được xác định từ vectơ các biến ngẫu nhiên $L(\chi)$.

Khi tồn tại các mối quan hệ không gian giữa các biến ngẫu nhiên $L(\chi)$ trong trường ngẫu nhiên $\mathrm{D}$, dựa trên phương pháp trung bình trọng số phương pháp địa thống kê đặt ra bài toán xác định giá trị tin cậy nhất của biến ngẫu nhiên $\widetilde{L}\left(\chi_{P}\right)$ tại điểm $\mathrm{p}$ thuộc tập hợp $P^{e}$ theo công thức:

$$
\tilde{L}\left(\chi_{P}\right)=\lambda^{T} . L(\chi),
$$

Ở đây vectơ $\lambda$ có dạng:

$$
\lambda=\left[\begin{array}{l}
\lambda_{1} \\
\lambda_{2} \\
\lambda_{n}
\end{array}\right],
$$

thêm vào đó thành phần $\lambda_{i}(i=1,2, \ldots, n)$ của vectơ $\lambda$ là trọng số tương đối của biến ngẫu nhiên $L\left(\chi_{i}\right)$ thoả mãn điều kiện:

$$
\lambda^{T} \cdot e=1
$$

với vectơ

$$
e=\left[\begin{array}{l}
1 \\
1 \\
\cdot \\
1
\end{array}\right]_{n x 1} .
$$




\section{Nghiên cúu}

Trong tài liệu (Jin Li and Andrew D. Heap (2008)) đã trình bày phương pháp nội suy nghịch đảo khoảng cách theo công thức (5), ở đây

$$
\lambda_{i}=\frac{\left(S_{i, P}\right)^{\alpha}}{\sum_{j=1}^{n} \frac{1}{\left(S_{j, p}\right)^{\alpha}}},
$$

a là số mũ, còn $S_{i . P}$ là khoảng cách từ điểm i thuộc tập hợp $Q$ đến điểm $p$ thuộc tập hợp $P^{e}$

Chúng ta nhận thấy rằng các thành phần $\lambda_{i}(i=1,2, \ldots, n)$ của vectơ $\lambda$ hoàn toàn thỏa mãn điều kiện (6). Điều kiện (6) là điều kiện ràng buộc đối với vectơ 2 để nhận được đánh giá tin cậy nhất của biến ngẫu nhiên $\widetilde{L}\left(\chi_{P}\right)$ theo công thức (5). Điều kiện (6) cũng được sử dụng rộng rãi trong phương pháp nội suy kriging (xem các tài liệu Naser El - Sheimy (1999)). Cressie N.A.C. (1993); Rao C.R., Toutenburg H. (1999); Marcin Ligas, Marek Kulczycki (2010).

Trong bài báo khoa học này chúng ta sẽ nghiên cứu hai phương pháp kriging và collocation nổi tiếng trong lý thuyết địa thống kê trên cơ sở đánh giá khả năng của chúng thỏa mãn hai điều kiện (1) và (2).

\section{Giải quyết vấn đề}

Bây giờ chúng ta ký hiệu giá trị trung bình xác xuất $\bar{L}\left(\chi_{P}\right)$ của biến ngẫu nhiên tin cậy nhất $\widetilde{L}\left(\chi_{P}\right)$ tại điểm $p \in P^{e}$ thỏa mãn điều kiện (3) bằng $\mu\left(\chi_{p}\right)$. Lưu ý (4) từ điều kiện không xê dịch (1) chúng ta có:

$$
E\left[\widetilde{L}\left(\chi_{P}\right)-\bar{L}\left(\chi_{P}\right)\right]=E\left[\lambda^{T} \cdot L(\chi)\right]-\mu\left(\chi_{p}\right)=0
$$

và từ đây nhận được điều kiện không xê dịch ở dạng:

$$
\bar{L}\left(\chi_{p}\right)=\lambda^{T} \Omega(\chi)=\mu\left(\chi_{p}\right),
$$

Điều kiện (7) cũng là điều kiện không xê dịch đối với biến ngẫu nhiên tin cậy nhất $\tilde{L}\left(\chi_{P}\right)$ và được sử dụng để xác định vectơ trend $\Omega(\chi)$. Do vectơ trend $\Omega(\chi)$. (4) được xác định từ vectơ các biến ngẫu nhiên $L(\chi)$, nên khi nhận nó làm vectơ các giá trị gần đúng xác suất của các biến ngẫu nhiên $L\left[\chi_{i}\right](i=1,2, \ldots, n)$ trong tập hợp $Q \in D$, lưu ý (7) chúng ta biến đổi công thức (5) về dạng:

$$
\widetilde{L}\left(\chi_{P}\right)=\mu\left(\chi_{p}\right)+\lambda^{T} .\{L(\chi)-\Omega(\chi)\} .
$$

Khi ký hiệu vectơ độ lệch của các biến ngẫu nhiên $L(\chi)$ so với các giá trị trend $\Omega(\chi)$. tương ứng dưới dạng:

$$
Z(\chi)=L(\chi)-\Omega(\chi)
$$

và vectơ độ lệch xác suất nhất tại điểm $p \in P^{e}$ dưới dạng: 


$$
\tilde{Z}\left(\chi_{p}\right)=\lambda^{T} \cdot Z(\chi),
$$

công thức (8) được biểu diễn dưới dạng mới:

$$
\tilde{L}\left(\chi_{P}\right)=\mu\left(\chi_{p}\right)+\lambda^{T} . Z(\chi)=\mu\left(\chi_{p}\right)+\widetilde{Z}\left(\chi_{p}\right) .
$$

Từ $(9),(10)$ chúng ta thấy rằng các độ lệch $Z(\chi)$ và $\widetilde{Z}(\chi)$ thỏa mãn tính chất:

$$
E\{Z(\chi)=E[\tilde{Z}(\chi)]=0 .
$$

Tiếp theo, chúng ta sẽ nghiên cứu hai phương pháp kriging và collocation để xác định vectơ $\lambda^{T}$ trong các công thức (8) hoặc (11).

\subsection{Phương pháp kriging}

Giả thiết rằng trên tập hợp gồm $n$ điểm $Q$ chúng ta đã xác định được $n$ giá trị biến ngẫu nhiên $L\left[\chi_{i}\right](i=1,2, \ldots ., n)$. Bài toán được đặt ra là cần xác định biến ngẫu nhiên $\widetilde{L}\left(\chi_{P}\right)(5)$ tại điểm $p \in P^{e}$ sao cho thỏa mãn các điều kiện (1) và (2). Chúng ta thay điều kiện không xê dịch (1) bằng điều kiện không xê dịch (7) với mục đích xác định vectơ trend $\Omega(\chi)$. (4). Khi đó các thành phần $\mu\left(\chi_{i}\right)(i=1,2, \ldots, n)$ được biểu diễn dưới dạng mô hình đa thức $\mu\left(\chi_{i}\right)=F_{1 x q}\left(\chi_{i}\right) \cdot \beta_{q x 1}$, ở đây $F_{1 x q}$ - vectơ các hệ số của mô hình, $\beta_{q x 1}$ là vectơ tham số của mô hình, q là bậc của đa thức (xem các tài liệu Goovaerts P. (1997); Olea R. A. (1999); Marcin Ligas, Marek Kulczycki (2014)). Đối với n biến ngẫu nhiên $L\left[\chi_{i}\right](i=1,2, \ldots . ., n)$ trong tập hợp $Q \in D$, chúng ta ký hiệu ma trận

$$
\bar{F}_{n x q}(\chi)=\left[\begin{array}{l}
F\left(\chi_{1}\right) \\
F\left(\chi_{2}\right) \\
\cdot \\
F\left(\chi_{n}\right)
\end{array}\right],
$$

đối với vectơ các biến ngẫu nhiên $L(\chi)$ chúng ta lập hệ phương trình số cải chính $V(\chi)=\bar{F} . \beta-L(\chi)$ và sau khi giải hệ phương trình này theo phương pháp bình phương nhỏ nhất, chúng ta nhận được vectơ $\$$ tức vectơ trend $\Omega(\chi)$ (4) đã được xác định. Khi đó điều kiện không xê dịch (7) được biến đổi về dạng:

$$
\lambda^{T} \cdot \bar{F}=F\left(\chi_{p}\right) .
$$

Bây giờ, để xác định vectơ $\lambda$ cần sử dụng điều kiện (2). Khi lưu ý $Z\left(\chi_{p}\right)$ là độ lệch ngẫu nhiên của giá trị gần đúng $\bar{L}\left(\chi_{P}\right)$ so với giá trị $\widetilde{L}\left(\chi_{P}\right)$ từ $(7)$ chúng ta biểu diễn giá trị gần đúng $\bar{L}\left(\chi_{P}\right)$ dưới dạng:

$$
\bar{L}\left(\chi_{p}\right)=\mu\left(\chi_{p}\right)+Z\left(\chi_{p}\right) .
$$

Lưu ý (11), (13), điều kiện (2) có dạng: 


$$
\begin{aligned}
& \Phi(\lambda)=E\left[\widetilde{L}\left(\chi_{P}\right)-L\left(\chi_{P}\right)\right]^{2}=E\left[\mu\left(\chi_{P}\right)+\lambda^{T} \cdot Z(\chi)-\mu\left(\chi_{P}\right)-Z\left(\chi_{P}\right)\right]^{2}= \\
& =E\left[\lambda^{T} \cdot Z(\chi)-Z\left(\chi_{P}\right)\right]^{2}=\operatorname{Var}\left[\lambda^{T} \cdot Z(\chi)\right]+\operatorname{Var}\left[Z\left(\chi_{P}\right)\right]-2 \cdot \operatorname{Cov}\left[\lambda^{T} \cdot Z(\chi) \cdot Z\left(\chi_{P}\right)\right]= \\
& =\lambda^{T} \cdot C_{Q} \lambda+C(0)-2 \cdot \lambda^{T} \cdot C_{Q \bar{P}}
\end{aligned}
$$

ở đây $C_{Q}=C_{Z(\chi) Z(\chi)}$ là ma trận hiệp phương sai của các biến ngẫu nhiên $Z(\chi)$ trong tập hợp $\mathrm{Q}, \quad C_{Q^{p}}=C_{Z(\chi): Z\left(\chi_{P}\right)}$ là ma trận hiệp phương sai của các biến ngẫu nhiên $Z(\chi)$ trong tập hợp $Q$ và các biến ngẫu nhiên $Z\left(\chi_{P}\right)$ trong tập hợp $\mathrm{P}^{\mathrm{e}}, \mathrm{C}(0)=\operatorname{Var}\left(Z\left(\chi_{P}\right)\right)$ là hàm ngẫu nhiên (phương sai của vectơ $Z\left(\chi_{P}\right)$ ).

Để tìm cực tiểu của hàm (15) dưới các điều kiện (6), (13) chúng ta lập hàm Lagrange:

$$
\Phi(\lambda, k)=\lambda^{T} \cdot C_{Q} \cdot \lambda+C(0)-2 \cdot \lambda^{T} \cdot C_{Q^{P}}-2 \cdot k_{1} \cdot\left(\lambda^{T} \cdot e-1\right)-2 \cdot\left(\lambda^{T} \cdot \bar{F}-F\left(\chi_{p}\right)\right) \cdot k_{2} \rightarrow \min ,
$$

ở đây $\mathrm{k}$ - nhân tử Lagrange.

Lần lượt lấy đạo hàm hàm trên theo các biến $\boldsymbol{\lambda}, \mathrm{k}_{1}, \mathrm{k}_{2}$ và cho bằng 0 , chúng ta nhận được hệ phương trình chuẩn:

$$
\begin{cases}C_{Q} \cdot \lambda+k_{1} \cdot e+\bar{F} \cdot k_{2} & =C_{Q P e}, \\ e^{T} \lambda & =1, \\ \bar{F}^{T} \cdot \lambda & =F^{T}\left(\chi_{p}\right) .\end{cases}
$$

Sau khi giải hệ phương trình chuẩn (16), chúng ta tìm được các ẩn $\lambda_{1}, \lambda_{2}, \ldots, \lambda_{n}$ và $\mathrm{k}$. Từ công thức (5) hoặc (11) chúng ta xác định được giá trị tin cậy nhất $\widetilde{L}\left(\chi_{P}\right)$ của biến ngẫu nhiên cần tìm tại điểm theo phương pháp kriging thông thường.

\subsection{Phương pháp collocation}

Phương pháp collocation phương sai được đề xuất trong các tài liệu (Heiskanen $A$. W., Moritz H. (1967); Moritz $H$. (1980)) với mục đích xác định giá trị xác suất của độ lệch $\widetilde{L}\left(\chi_{P}\right)(10)$, chứ không phải giá trị xác suất của biến ngẫu nhiên $\widetilde{L}\left(\chi_{P}\right)$ của điểm $p \in P^{e}$ Chúng ta lưu ý rằng giá trị $\widetilde{Z}\left(\chi_{p}\right)$ và vectơ các độ lệch $Z(\chi)(9)$ đều thỏa mãn điều kiện (12).

Lưu ý công thức (10), chúng ta ký hiệu vectơ sai số $\varepsilon=Z\left(\chi_{p}\right)-\widetilde{Z}\left(\chi_{p}\right)=Z\left(\chi_{p}\right)-\lambda^{T} . Z(\chi)$. Khi chuyển sang sai số trung phương của dự báo, chúng ta có:

$$
\begin{aligned}
& D_{\widetilde{Z}\left(\chi_{p}\right)}=m_{\widetilde{Z}\left(\chi_{p}\right)}^{2}=E\left[\varepsilon^{2}\right]=E\left[\left(Z\left(\chi_{p}\right)-\lambda^{T} \cdot Z(\chi)\right)^{2}\right]= \\
& =\operatorname{Var}\left(Z\left(\chi_{p}\right)\right)+\operatorname{Var}\left(\lambda^{T} \cdot Z(\chi)\right)-2 \cdot \operatorname{Cov}\left(Z\left(\chi_{p}\right) \cdot \lambda^{T} \cdot Z(\chi)\right)= \\
& =C(0)+\lambda^{T} \cdot C_{Q^{\prime}} \cdot \lambda-2 \cdot \lambda^{T} \cdot C_{Q^{p e}},
\end{aligned}
$$


ở đây $\mathrm{C}(0)=\operatorname{Var}\left(Z\left(\chi_{P}\right)\right)$ là hàm ngẫu nhiên (phương sai của vectơ $Z\left(\chi_{P}\right)$. Bây giờ chúng ta phải tìm vectơ $\lambda^{T}$ sao cho sai số trung phương $m_{\widetilde{Z}\left(\chi_{p}\right)}^{2}$ đạt cực tiểu.
Khi đó lấy đạo hàm (17) theo $\lambda^{T}$ và cho bằng 0 , chúng ta nhận được

$$
\frac{\partial m_{\widetilde{Z}\left(\chi_{p}\right)}^{2}}{\partial \lambda}=2 \cdot C_{Q} \cdot \lambda-2 \cdot C_{Q P}=0
$$

và từ đây suy ra công thức:

$$
\lambda^{T}=C_{P Q} \cdot C_{Q}^{-1}
$$

Như vậy công thức (10) có dạng:

$$
\widetilde{Z}\left(\chi_{p}\right)=C_{P Q} \cdot C_{Q}^{-1} \cdot Z(\chi) \text {. }
$$

Công thức đánh giá (19) theo phương pháp collocation đã được cho trong tài liệu (Moritz H. (1980)).

Mặc dù phương pháp collocation được sử dụng rộng rãi trong trắc địa vật lý, nhưng chúng ta cũng cần thiết phải bình luận. Như đã nêu ở trên, phương pháp collocation được đề xuất với mục đích xác định giá trị xác suất của độ lệch $\widetilde{Z}\left(\chi_{p}\right)$, chứ không phải giá trị của biến ngẫu nhiên tin cậy nhất $\widetilde{L}\left(\chi_{P}\right)$ của điểm $p \in P^{e}$

Nếu vectơ độ lệch $Z(\chi)$ trong (19) được xác định theo công thức (9), ở đây vectơ trend $\Omega(\chi)$ được xác định từ n biến ngẫu nhiêr $\left.L\left[\chi_{i}\right]^{\prime} i=1,2, \ldots, n\right)$ trong tập hợp $Q \in D$, thì đánh giá giá trị của biến ngẫu nhiên tin cậy nhất $\widetilde{L}\left(\chi_{P}\right)$ của điểm $p \in P^{e}$ theo công thức (11) sẽ là đánh giá không xê dịch. Trong trường hợp ngược lại, đánh giá $\widetilde{L}\left(\chi_{P}\right)$ sẽ bị xê dịch, tức không gần nhất với giá trị thực của nó.

Ở Việt Nam, việc sử dụng các giá trị dị thường trọng lực được tính toán từ mô hình EGM2008 để làm các giá trị gần đúng xác suất $\mu\left(\chi_{i}\right)$ của vectơ trend $\Omega(\chi)$ trong quá trình nội suy các giá trị dị thường trọng lực mặt đất trong bài toán xây dựng cơ sở dị thường trọng lực quốc gia không đảm bảo tính chất (4), tức vectơ trend $\Omega(\chi)$ không được xác định từ các giá trị dị thường trọng lực mặt đất do các dữ liệu trọng lực mặt đất ở Việt Nam không tham gia xác định các hệ số điều hoà của mô hình EGM2008. Vì lý do này chúng ta sẽ nhận được các giá trị dị thường trọng lực được nội suy $\widetilde{L}\left(\chi_{P}\right)$ bị xê dịch, tức chúng hoàn toàn có khả năng bị sai lệch lớn so với các giá trị thực của chúng. Các kết quả thực nghiệm cho thấy ở khu vực vùng núi Việt Nam, các dị thường trọng lực từ EGM2008 bị sai lệch so với các giá trị dị thường trọng lực mặt đất đến hơn 20 mGal.

Ngoài ra, trong lý thuyết của phương pháp collocation trung phương chưa tính đến điều kiện (6) của mô hình bài toán nội suy (5) hoặc (11). Điều này giải thích sự khác nhau của vectơ $\lambda^{T}(18)$ theo phương pháp collocation so với của vectơ $\lambda^{T}$ được giải từ hệ (16) theo phương pháp kriging. Đây là vấn đề đang đặt ra để nghiên cứu hoàn thiện phương pháp collocation. 


\section{Kết luận}

Các phương pháp nội suy được xây dựng dựa trên phương pháp bình phương nhỏ nhất đều phải tuân thủ chặt chẽ các điều kiện (1), (2) của định lý Gauss - Markov với điều kiện các biến ngẫu nhiên $L\left[\chi_{i}\right](i=1,2, \ldots, n)$ tham gia tính toán không được chứa các sai số hệ thống và các sai số thô. Bên cạnh đó, các giá trị gần đúng xác suất $\mu\left(\chi_{i}\right)$ của biến ngẫu nhiên $L\left[\chi_{i}\right](i=1,2, \ldots, n)$ của vectơ trend $\Omega(\chi)$ trong công thức (8) hoặc (9) phải được tính toán từ chính các biến ngẫu nhiên $L\left[\chi_{i}\right](i=1,2, \ldots, n)$ để đảm bảo thỏa mãn điều kiện không xê dịch (7). Các điều nêu ở trên mới đảm bảo vectơ các độ lệch $Z(\chi)(9)$ và đánh giá (19) theo phương pháp collocation thỏa mãn điều kiện (12). Tuy nhiên việc không sử dụng điều kiện (6) của mô hình bài toán nội suy làm đánh giá theo phương pháp collocation không tương ứng với mô hình của bài toán nội suy (5) hoặc (11). O

\section{Tài liệu tham khảo}

[1]. Cressie N.A.C. (1993). Statistics for spatial data. John Wiley \& Sons, New York. 900p.

[2]. Goovaerts P. (1997). Geostatistics for natural resources evaluation. New York, Oxford University Press, $483 \mathrm{p}$.

[3]. Hà Minh Hòa (2014). Phương pháp xử lý toán học các mạng lưới trắc địa quốc gia. Nhà Xuất bản Khoa học và Kỹ thuật, 244 trg. Hà Nội - 2014.

[4]. Heiskanen W.A. and Moritz H. (1967). Physical Geodesy. W.H. Freeman, San Francisco, California, $364 \mathrm{p}$.

[5]. Jin Li and Andrew D. Heap (2008). A Review of Spatial Interpolation Methods for Environmental Scientists. Geoscience Australia Record 2008/23, 137 p.

[6]. Kolmogorov A.N. (1933). Foundations of the Theory of Probability. Chelsea Publishing, New York.

[7]. Marcin Ligas, Marek Kulczycki (2010). Simple spatial prediction - least squares prediction, simple kriging, and conditional expectation of normal vector. J. Geodesy And Cartography, Vol. 59, N02, pp. 69-81. Polish Academy of Sciences.

[8]. Marcin Ligas, Marek Kulczycki (2014). Kriging approch for local height transformations. J. Geodesy And Cartography, Vol. 63, N01, pp. 25-37. Polish Academy of Sciences. Doi: 10.2478/geocart-2014-0002.

[9]. Moritz H. (1980). Advanced Physical Geodesy. Herbert Wichmann, Verlag, Karlsruhe, $500 \mathrm{p}$.

[10]. Naser El - Sheimy (1999). Digital Terrain Modelling, ENGO 573, Vol. 1. Geomatics Engineering Department, The University of Calgary, $18 \mathrm{p}$.

[11]. Olea R. A. (1999). Geostatistics for engineers and earth scientists: Norell, Mass., Boston, Kluwer Academic Publishers, 313 p.

[12]. Rao C.R., Toutenburg H. (1999). Linear models - least squares and alternatives. Springer, New York.

[13]. Wiener N. (1949). Extrapolation, Interpolation and Smoothing of Stationary Time Siries, MIT Press, Cambridge, MA.O

(Xem tiếp trang 24) 